\title{
Wushenziye Formula Improves Skeletal Muscle Insulin Resistance in Type 2 Diabetes Mellitus via PTP1B-IRS1-Akt-GLUT4 Signaling Pathway
}

\author{
Chunyu Tian, ${ }^{1,2}$ Hong Chang, ${ }^{1,2}$ Xiaojin La, ${ }^{1,2}$ and Ji-an $\mathrm{Li}^{1,2}$ \\ ${ }^{1}$ North China University of Science and Technology, Tangshan 063210, China \\ ${ }^{2}$ Pharmacology Analysis Key Laboratory for Prevention and Treatment of Diabetes of Traditional Chinese Medicine in Hebei Province, \\ Tangshan 063210, China
}

Correspondence should be addressed to Chunyu Tian; tcy4479@sina.com

Received 8 September 2017; Revised 25 November 2017; Accepted 4 December 2017; Published 31 December 2017

Academic Editor: Akhilesh K. Tamrakar

Copyright (C) 2017 Chunyu Tian et al. This is an open access article distributed under the Creative Commons Attribution License, which permits unrestricted use, distribution, and reproduction in any medium, provided the original work is properly cited.

\begin{abstract}
Background. Wushenziye formula (WSZYF) is an effective traditional Chinese medicine in the treatment of type 2 diabetes mellitus (T2DM). Aim. This study aimed to identify the effects and underlying mechanisms of WSZYF on improving skeletal muscle insulin resistance in T2DM. Methods. An animal model of T2DM was induced by Goto-Kakizaki diabetes prone rats fed with high fat and sugar for 4 weeks. Insulin resistance model was induced in skeletal muscle cell. Results. In vivo, WSZYF improved general conditions and decreased significantly fasting blood glucose, glycosylated serum protein, glycosylated hemoglobin, insulin concentration, and insulin resistance index of T2DM rats. In vitro, WSZYF enhanced glucose consumption in insulin resistance model of skeletal muscle cell. Furthermore, WSZYF affected the expressions of molecules in regulating T2DM, including increasing the expressions of p-IRS1, p-Akt, and GLUT4, reducing PTP1B expression. Conclusion. These findings displayed the potential of WSZYF as a new drug candidate in the treatment of T2DM and the antidiabetic mechanism of WSZYF is probably mediated through modulating the PTP1B-IRS1-Akt-GLUT4 signaling pathway.
\end{abstract}

\section{Introduction}

Diabetes mellitus characterized by deregulation of glucose and lipid metabolism seriously affects human health. It affected an estimated 366 million people in 2011 and the number is projected to be 600 million by the year 2035 [1]. Type 2 diabetes mellitus (T2DM), one of the most common types of diabetes with the characteristics of insensitivity to insulin, has attracted great attention [2]. Insulin resistance (IR), an impaired biological response to insulin, is the pathological basis of T2DM. It refers to the decreased sensitivity of tissues to insulin, resulting in the reduction in glucose uptake and utilization [3]. In the body, skeletal muscle is the main target organ that consumes glucose and completes approximately $80 \%$ of the postprandial glucose intake and consumption caused by insulin stimulation [4, 5]. However, under the condition of IR, insulin-stimulated glucose disposal in skeletal muscle is severely damaged and could not respond to insulin properly. This leads to a defect in the insulin signaling pathway in muscle and elevating blood glucose level, which is a key feature of IR in T2DM. Therefore, skeletal muscle was selected as a therapeutic target of WSZYF in the battle against T2DM.

The main proteins in the phosphatidylinositol 3-kinase (PI3K) signaling pathway, including PI3K, Akt, and glucose transporter type 4 (GLUT4), play important roles in insulin signaling transduction. The abnormality of this pathway is the major cause of T2DM [6]. The signaling cascade is triggered when insulin connects with the membrane receptor of target cells. Insulin receptor substrate-1 (IRS-1) phosphorylated by the activated insulin receptor leads to PI3K and Akt activation [7]. GLUT4, downstream of PI3K, is the pivotal protein in controlling glucose uptake and glycogen metabolism [8]. In T2DM, not only is the sensitivity of skeletal muscle to insulin abated, but also the expression of GLUT4 is decreased, which decreases the glucose uptake and utilization and raises blood glucose level $[9,10]$. Moreover, the lack activity of IRS-1 leads to the reduced phosphorylation of PI3K and 
thereby decreased GLUT4 expression in skeletal muscle cells, resulting in IR [11]. Thus, the modulation of IRS1-Akt-GLUT4 signaling pathway is quite associated with the treatment of IR.

Protein tyrosine phosphatase-1B (PTP1B), which leads to the dephosphorylation of insulin receptor, has the negative regulation in insulin signal transduction [12]. Studies [12-14] have demonstrated that inhibition of PTP1B could result in the phosphorylation of insulin receptor and insulin receptor substrates (IRS), thus improving insulin sensitivity and decreasing blood glucose level. Therefore, targeting on PTP1B may be a novel approach for the treatment of T2DM.

Traditional Chinese medicine has made significant contributions to the prevention and treatment of T2DM. WSZYF is an effective compound in the treatment of T2DM in clinic. It consists of four Chinese medicines, including Radix Polygoni Multiflori Preparata, Mori fructus, Mori folium, and Cassiae semen. It was testified that components in WSZYF, like resveratrol and 2-styrene glucoside $[15,16]$, had the effects on improving insulin sensitivity. The antidiabetic effect and underling mechanism of WSZYF have not been clearly explained. In this study, a T2DM rat model and an IR model of skeletal muscle cell were established to investigate whether WSZYF could improve glucose metabolism and IR or not and how to regulate PTP1B-IRS1-Akt-GLUT4 signaling pathway by WSZYF.

\section{Materials and Methods}

2.1. Materials. Insulin assay kit was obtained from Millipore (USA). Dulbecco's modified Eagle's medium (DMEM) and fetal bovine serum (FBS) were from Gibco (USA). Metformin was purchased from Squibb Pharmaceutical (Shanghai, China). Blood sugar meter and blood sugar test paper were purchased from Sannuo Biosensors (China). Assay kits of glycosylated serum protein and glycosylated hemoglobin were from Nanjing Jiancheng Bioengineering Institute (Nanjing, China). The primary antibodies against PTP1B, p-Akt (phospho S473), GLUT4, and GAPDH were from Bioworld Technology (USA) and antibody against p-IRS1 (phospho Y612) was from Abcam (USA). The secondary antibodies were purchased from Beyotime Biotechnology (China). The herbs in WSZYF were purchased from Tongrentang Pharmacy (Tangshan, China).

2.2. Animals and Experimental Design. All the procedures were conformed to the Guide for the Care and Use of Laboratory Animals published by the National Institutes of Health (NIH Publications number 85-23, revised 1996). Goto-Kakizaki (GK) rats weighing 200 to $300 \mathrm{~g}$ were purchased from Shanghai Slack Animal Center (certificate number SCXK (Shanghai) 2012-0002). Rats were raised in specific pathogen-free (SPF) room in North China University of Science and Technology. After one-week acclimation, rats were fed with high glucose and fat for 4 weeks to induce T2DM model. Rats with fasting blood glucose $\geqslant 11.1 \mathrm{mmol} / \mathrm{L}$ and blood glucose $\geqslant 16.7 \mathrm{mmol} / \mathrm{L}$ were randomly divided into five groups: model group $(n=6$, oral administration of equivalent volume of normal saline), metformin group ( $n=6$, oral administration of metformin, $85 \mathrm{mg} / \mathrm{kg} /$ day),
WSZYF low-dose group (WSZYF (L), $n=6$, oral administration of WSZYF, $300 \mathrm{mg} / \mathrm{kg} /$ day), WSZYF medium-dose group (WSZYF (M), $n=6$, oral administration of WSZYF, $600 \mathrm{mg} / \mathrm{kg} / \mathrm{day}$ ), and WSZYF high-dose group (WSZYF $(\mathrm{H})$, $n=6$, oral administration of WSZYF, $1200 \mathrm{mg} / \mathrm{kg} /$ day). The Wistar rats orally given equivalent volume of normal saline served as the control group. All the rats were raised for 8 weeks. At the end of the study, general conditions were measured and indexes related to blood glucose and insulin were tested. Muscle tissues were stored at $-80^{\circ} \mathrm{C}$ for further analysis.

2.3. Body Weight, Food Intake, Water Intake, and Urine Output. The body weight, food intake, water intake, and urine output of the rats were monitored at the time points of $0,1,4$, and 8 weeks and the data of 8 weeks were selected in this study. For urine output, rats were put into the metabolic cage after fasting for 12 hours. Urine in 24 hours was collected. During this time, they were only provided with food but no water.

2.4. Blood Chemistry Assay. After eight weeks, blood samples were collected for blood chemistry measurements. Fasting blood glucose was measured by using blood sugar meter and blood sugar test paper. Glycosylated hemoglobin, glycosylated serum protein, and insulin were detected by kits analysis according to manufacturer's instruction. Insulin resistance was assessed by a homeostasis model assessment of IR index as previously described [17].

2.5. Glucose Concentration in the Cell Supernatant Analysis. Primary cell culture of skeletal muscle was performed as previous study [18]. Muscle tissue was dissected from the soleus and cut into small pieces at the size of $0.1 \mathrm{~cm}^{3}$ and washed 3 times with PBS and DMEM (mixed with 10\% FBS and $2 \%$ penicillin/streptomycin). Then the small pieces of muscle tissue were seeded in culture flasks which were upside down in in a humidified incubator with $5 \% \mathrm{CO}_{2}$ at $37^{\circ} \mathrm{C}$. After 2 hours, the culture flasks were right side up and 3-5 $\mathrm{ml}$ culture medium was added. The culture medium was renewed every three days, while cultures were monitored by daily observation under an inverted microscope (Olympus, Japan). Then the cells were purified by differential adherence and the second generation was used in the experiment. When the skeletal muscle cells reached $70 \%$ confluence, they were induced to differentiation by DMEM supplemented with $2 \%$ FBS, $100 \mathrm{U} / \mathrm{ml}$ penicillin, and $100 \mu \mathrm{g} / \mathrm{ml}$ streptomycin. The successful differentiation showed that most of the myocytes had differentiated into multinucleated myotubes and could be easily identified as muscle cells. Then cells were divided into several groups: control group, model group, WSZYF-L $(25 \mu \mathrm{g} / \mathrm{L})$ group, WSZYF-M $(100 \mu \mathrm{g} / \mathrm{L})$ group, and WSZYF-H $(400 \mu \mathrm{g} / \mathrm{L})$ group. In the model group, cells were washed three times with PBS and insulin $\left(5 \times 10^{-7} \mathrm{~mol} / \mathrm{L}\right)$ with DMEM $200 \mu \mathrm{l}$ was added for 12 hours. Then the supernatant was removed and DMEM was added for 24-hour incubation. Cells in the treatment groups were treated in a similar way, except that the culture media contained WSZYF in the later 24 hours. In the control group, fresh nutrient solution was changed at the time points of 12 and 24 hours. 


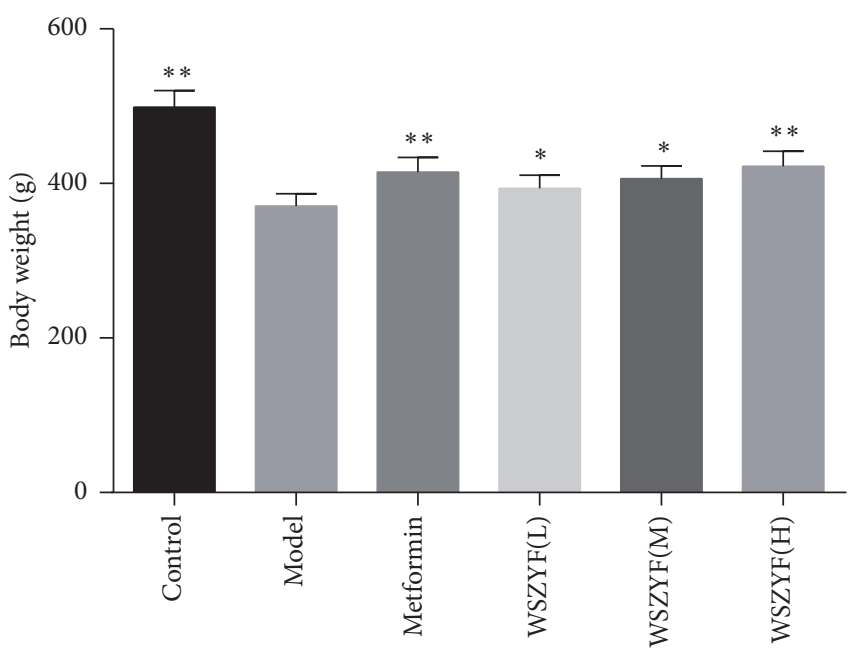

(a)

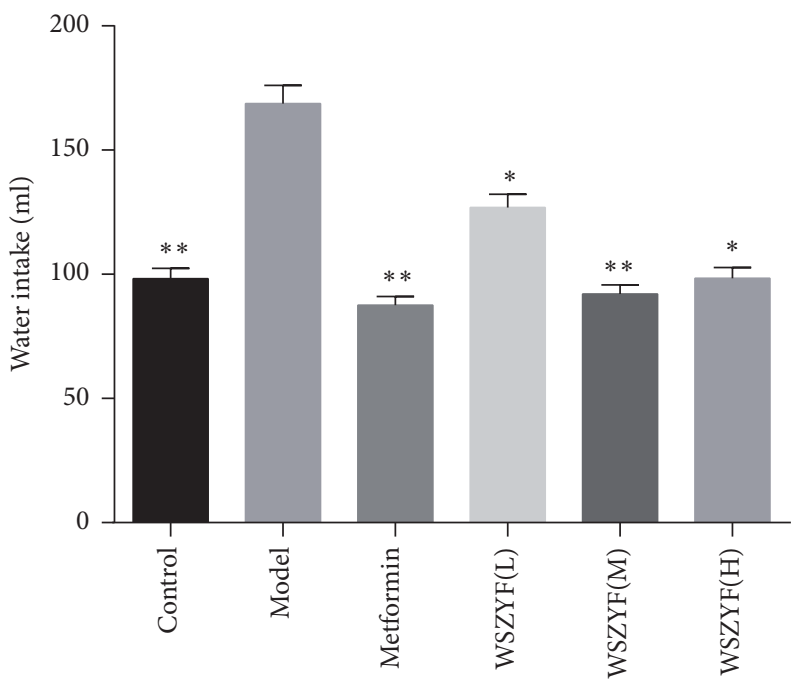

(c)

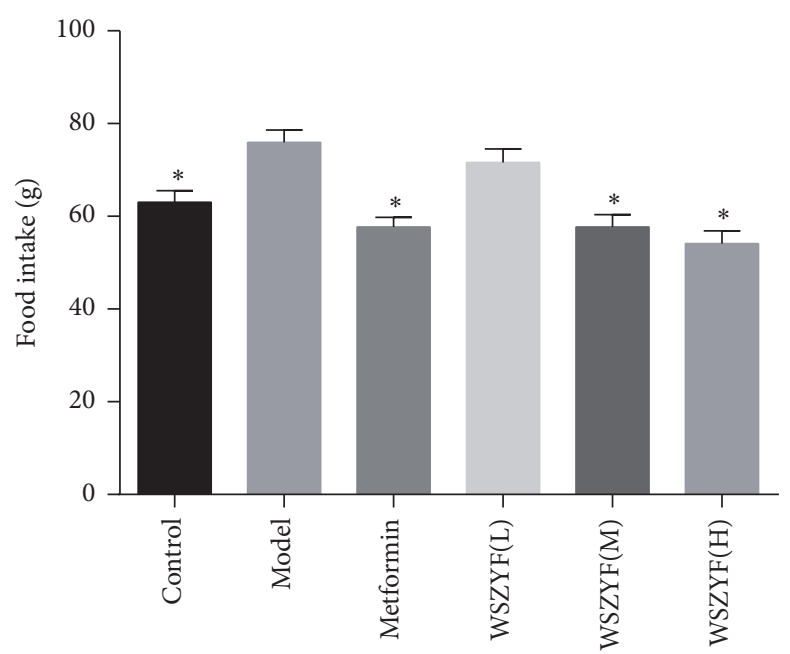

(b)

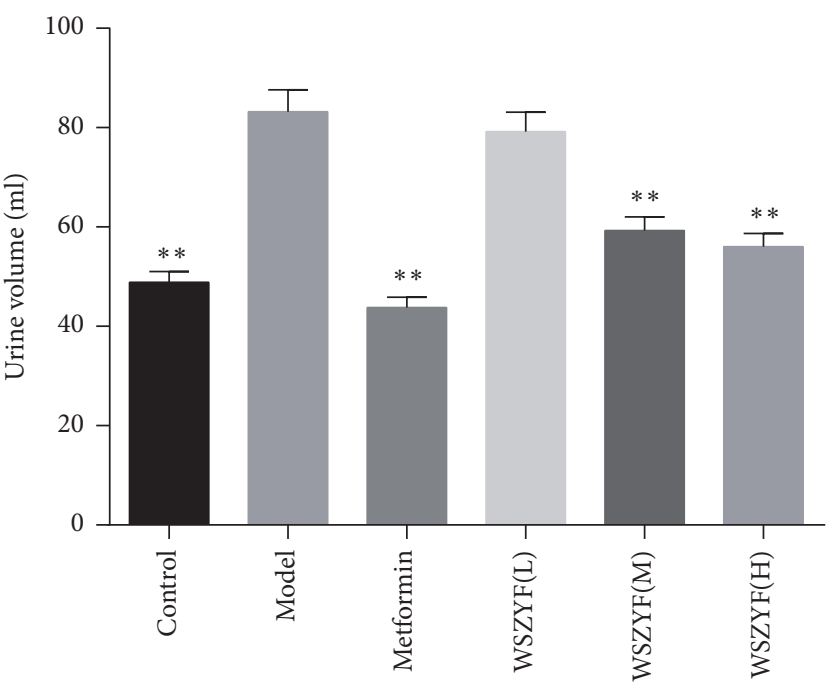

(d)

FIGURE 1: Effects of WSZYF on general conditions in T2DM rats. (a) Body weight, (b) food intake, (c) water intake, and (d) urine volume. ${ }^{*} P<0.05,{ }^{* *} P<0.01$ compared with model group.

2.6. Western Blotting Analysis on Proteins Related to Regulating Insulin Resistance. Proteins related to regulating insulin resistance were detected by in vivo study. The collected muscle tissues (120 mg) were prepared with RIPA buffer (PPLYGEN, China) and proteins were extracted according to the manufacturer's instruction. Protein contents were measured with BCA protein assay kit (PPLYGEN, China). After addition of loading buffer boiled for 5 minutes, tissue samples were separated by $10 \%$ SDS-PAGE and transferred to NC membranes (Millipore, Germany). After being blocked with 5\% nonfat dry milk for 2 hours, the membranes were incubated with different primary antibodies overnight at $4^{\circ} \mathrm{C}$. After washing with TBST three times, the membranes were incubated with HRP-conjugated secondary antibodies for 1 hour at room temperature. Washed three times with TBST, the proteins were detected with an enhanced chemiluminescence agent (GE, USA) and quantified by densitometry using an image analyzer (Bio-Rad, USA). Mouse anti-GAPDH monoclonal antibody served as an internal control.
2.7. Statistical Analysis. Data were expressed as the mean \pm standard deviation (SD). Statistical analysis was undertaken by one-way analysis of variance (ANOVA) and Dunnett's test. Differences between groups were considered as statistically significant when $P<0.05$.

\section{Results}

3.1. Effects of WSZYF on General Conditions in T2DM Rats. Food and water intake and urine output in the model group were significantly higher and body weight was lower than that of control group. Treatments of WSZYF $(\mathrm{M}, \mathrm{H})$ and metformin increased body weight and decreased food and water intake and urine output compared with the model group. These results indicated that WSZYF could ameliorate the general conditions of T2DM rats. Low dose of WSZYF had no significance in food intake or urine volume (Figure 1). 


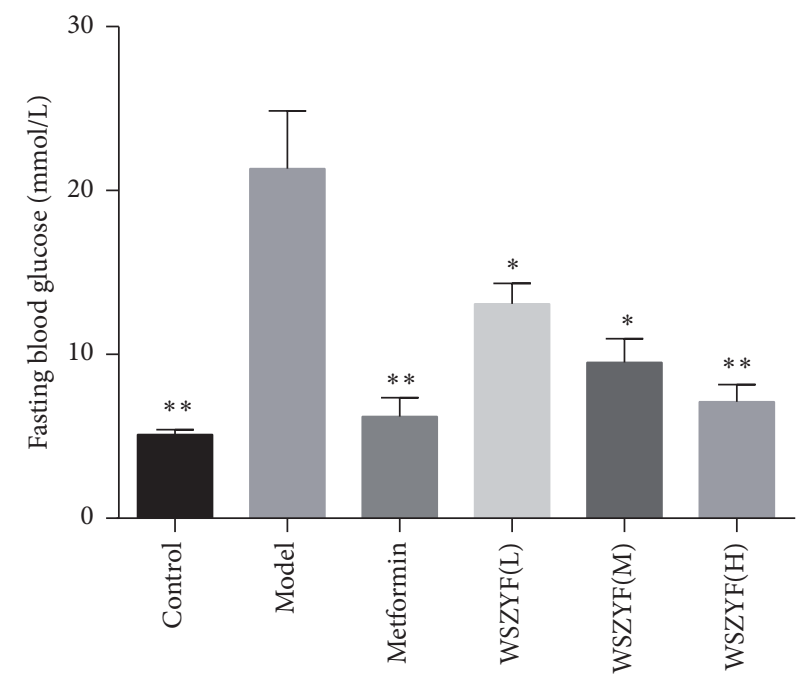

(a)

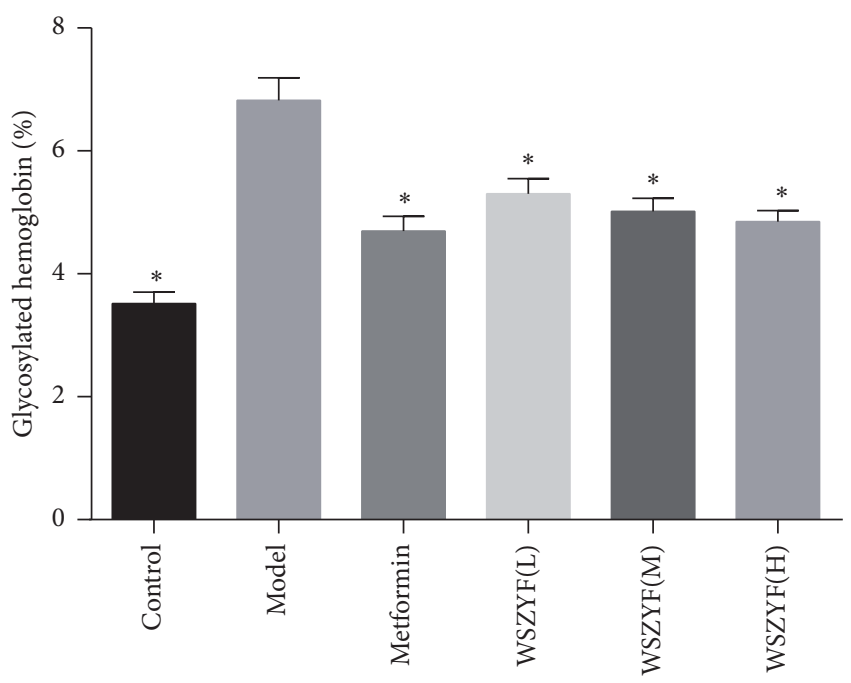

(b)

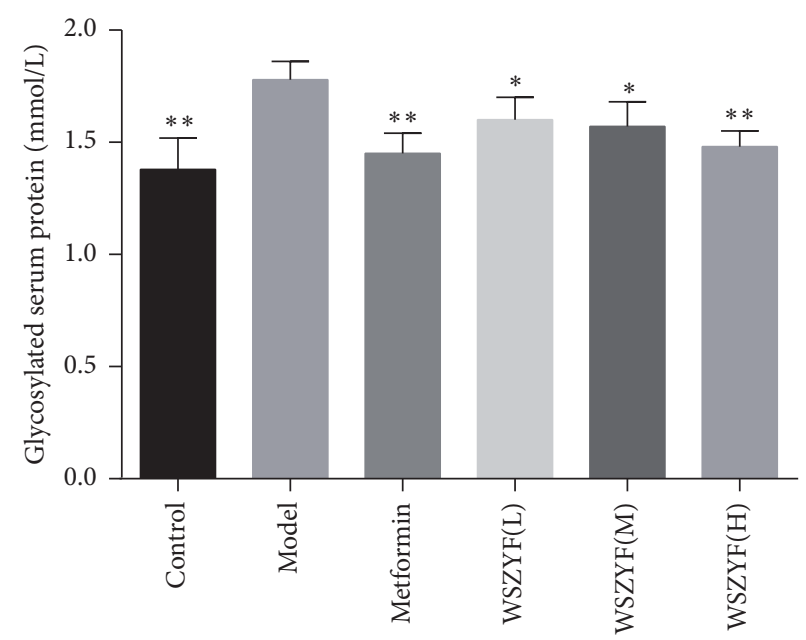

(c)

FIGURE 2: Effects of WSZYF on fasting blood glucose, glycosylated hemoglobin, and glycosylated serum protein. (a) Fasting blood glucose, (b) glycosylated hemoglobin, and (c) glycosylated serum protein. ${ }^{*} P<0.05,{ }^{* *} P<0.01$ compared with model group.

3.2. Effects of WSZYF on Glucose Metabolism Disorders. As shown in Figure 2, fasting blood glucose, glycosylated hemoglobin, and glycosylated serum protein increased significantly in T2DM model group. These results suggested that glucose metabolism was disrupted. Treatments of WSZYF and metformin lowered all the three indexes mentioned above, indicating that they could restore glucose metabolism disorders.

3.3. Effects of WSZYF on Insulin Sensitivity. Insulin plays a critical role in the maintenance of blood glucose homeostasis. Further studies displayed the effects of WSZYF on insulin sensitivity. Insulin concentration and IR index increased in model group, which proved the IR occurrence. After treatments, these two indicators were decreased to be normal, demonstrating that WSZYF had the ability to enhance insulin sensitivity (Figure 3).
3.4. Effects of WSZYF on Glucose Consumption. In order to test the effect of WZSYF on glucose uptake, an IR model of skeletal muscle induced by insulin stimulation was established. In Figure 4, the glucose concentration in the cell supernatant of model group was higher than that of control group, indicating that glucose consumption was decreased in model group. Treatments of WZSYF decreased glucose concentration in the cell supernatant, which proved WSZYF could enhance glucose uptake of skeletal muscle cells.

3.5. Effects of WSZYF on Expressions of Insulin Signaling Transduction-Related Proteins. We further investigated the mechanism of WSZYF in the regulation of insulin signal transduction. As shown in Figure 5, the expressions of P-IRS1, P-Akt, and GLUT4 decreased, whereas PTP1B expression increased in model group compared with control group, indicating that insulin signal transduction was disrupted in 


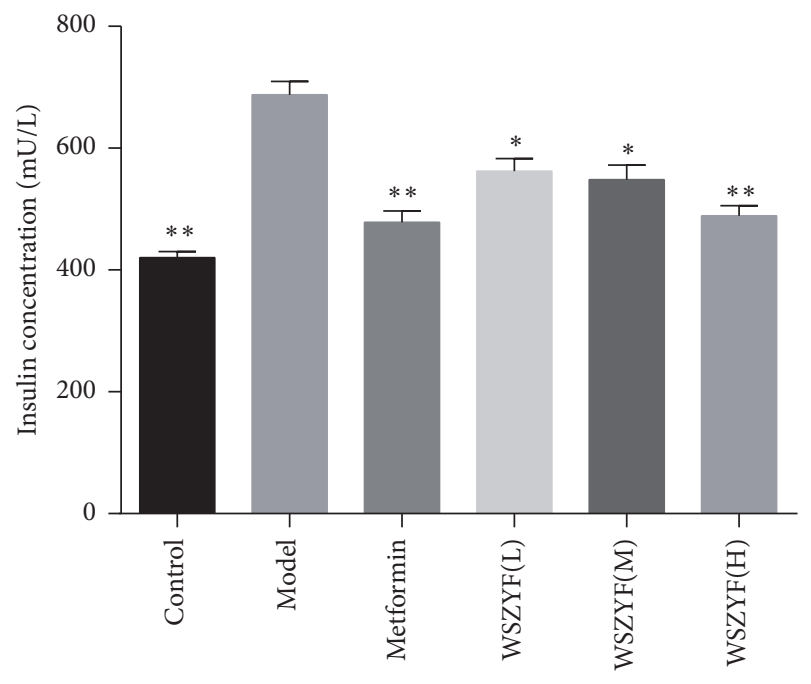

(a)

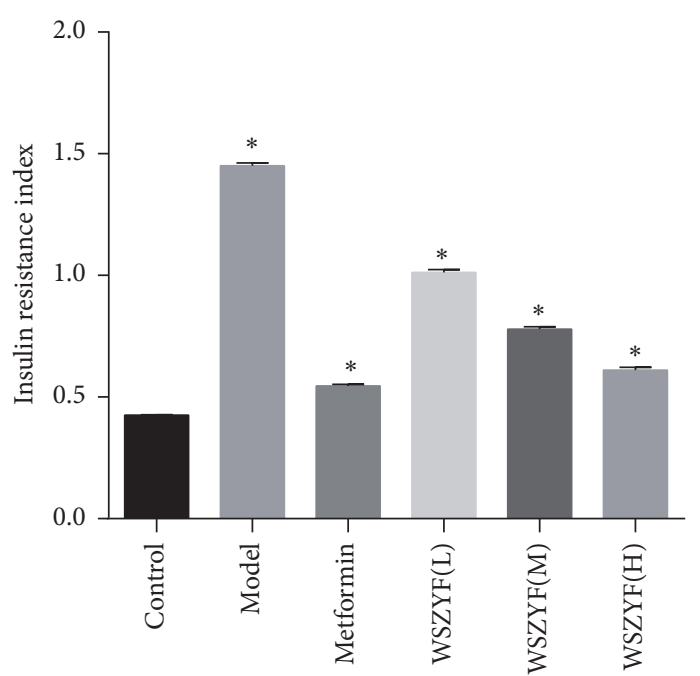

(b)

FIgURE 3: Effects of WSZYF on insulin and IR index. (a) Insulin concentration and (b) IR index. ${ }^{*} P<0.05$ and ${ }^{* *} P<0.01$ compared with model group.

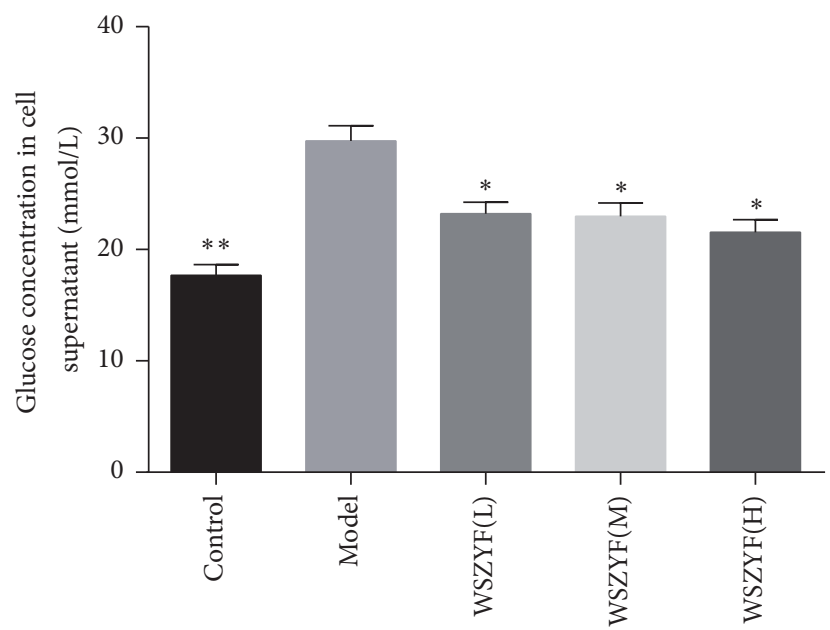

FIGURE 4: Effects of WSZYF on glucose consumption. ${ }^{*} P<0.05$ and ${ }^{* *} P<0.01$ compared with model group.

T2DM rats. Expressions of these proteins were reverted to normal levels in treatment of WSZYF and metformin. The results showed that the antidiabetic effect of WSZYF may be mediated by modulating PTP1B-IRS1-Akt-GLUT4 antiinsulin resistant signaling pathway.

\section{Discussion}

Insulin resistance of skeletal muscle has been shown to play a vital role in the pathogenesis of T2DM. In this study, we demonstrated that WSZYF could exert antidiabetic effect by improving IR in skeletal muscle. Further in vivo study suggested that the anti-insulin resistant effect of WSZYF was potentially triggered by regulating PTP1B-IRS1-Akt-GLUT4 signaling pathway.
In T2DM patients, insulin sensitivity decreases in most organs and tissues, accompanied with symptoms like hyperglycemia, hyperlipidemia, hyperinsulinism, and so on [19]. GK rats, a model of T2DM prone rats, were characterized by hyperglycemia, hyperinsulinemia, and IR [20]. Due to these symptoms being similar to T2DM in human, GK rats are widely used in T2DM research. In this study, GK rats fed with high fat and glucose for 4 weeks exhibited a series of T2DM symptoms, including increases of food and water intake as well as urine volume but reduction of body weight. Compared with model group, WSZYF could alleviate the above-mentioned symptoms and better effect was obtained in a higher dose. Moreover, WSZYF also observably decreased fasting blood glucose, glycosylated serum protein, glycosylated hemoglobin, insulin, and IR index compared with those in model group. Meanwhile, in vitro results showed that WSZYF could also enhance glucose consumption in IR model of skeletal muscle cells. All of these suggested that WSZYF could regulate glucose metabolism disorders and improve IR in T2DM rats.

There are three signaling pathways mentioned in insulin signal transduction pathways including PI3K pathway, mitogen activated protein kinase pathway, and $\mathrm{C}-\mathrm{Cbl}$ related protein pathway. Among them, PI3K signaling pathway is the classical one [21-24]. The normal transduction of PI3K signaling pathway is directly related to the normal metabolisms of glucose, fat, and protein. Meanwhile, any abnormalities of PI3K signaling pathway transduction may lead to IR [25]. In the normal process, IRS is phosphorylated when insulin combines with its receptor in the surface of skeletal muscle cell, followed by PI3K activation. The activated PI3K can catalyze phosphatidylinositol 4,5-bisphosphate (PIP2) to PIP3, which activates sequence downstream signaling factor Akt. Phosphorylated Akt translocates GLUT4 to the plasma membrane to uptake glucose into the muscle, which contributes to decreasing plasma glucose concentrations [26]. 


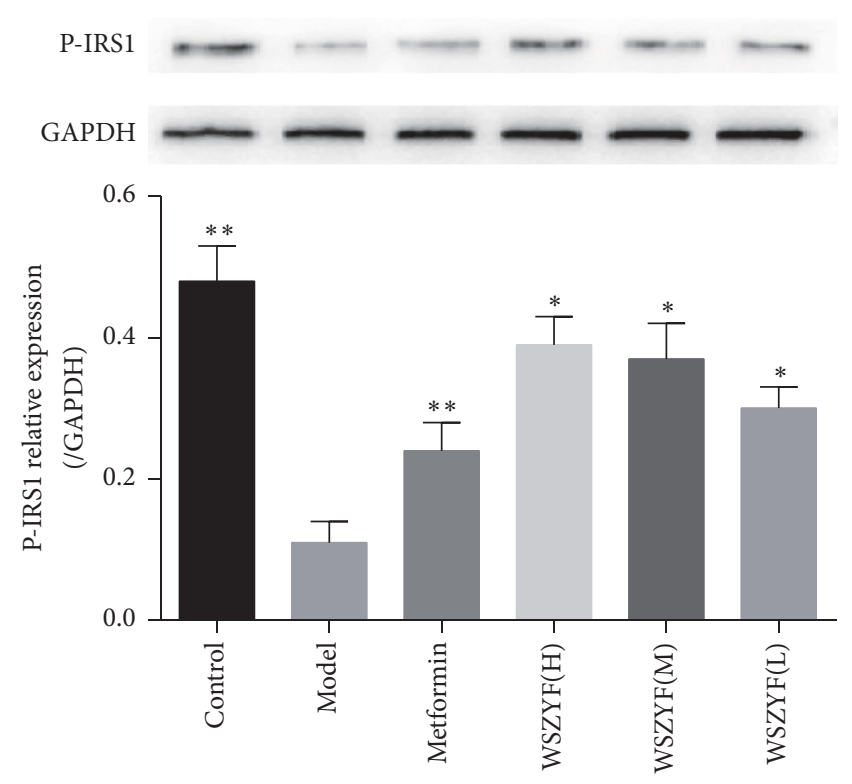

(a)
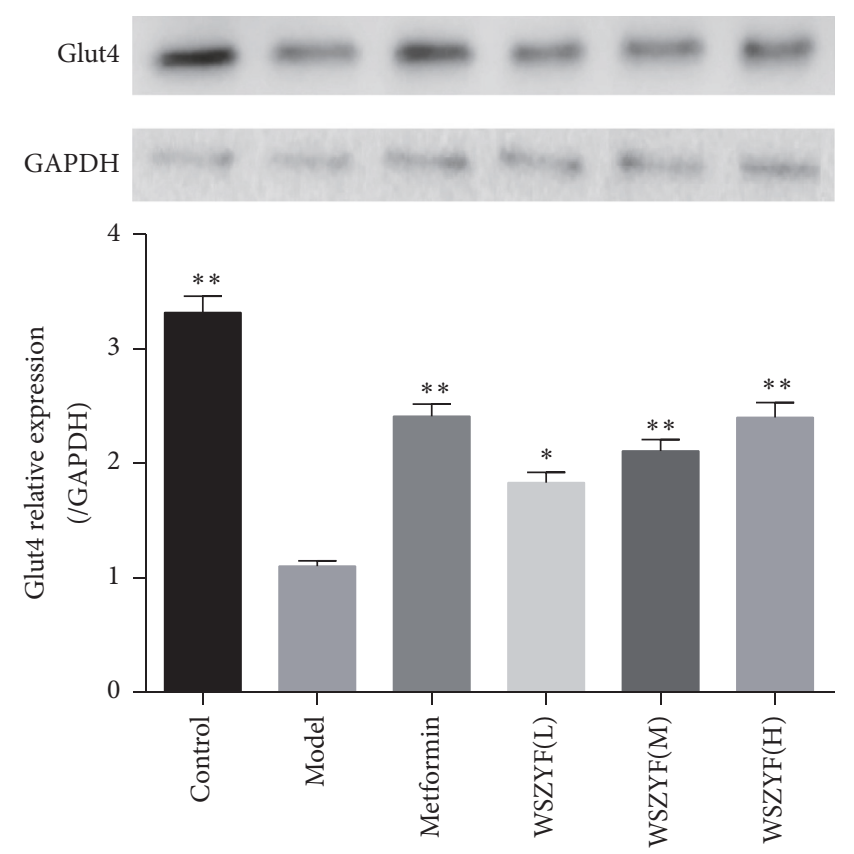

(c)

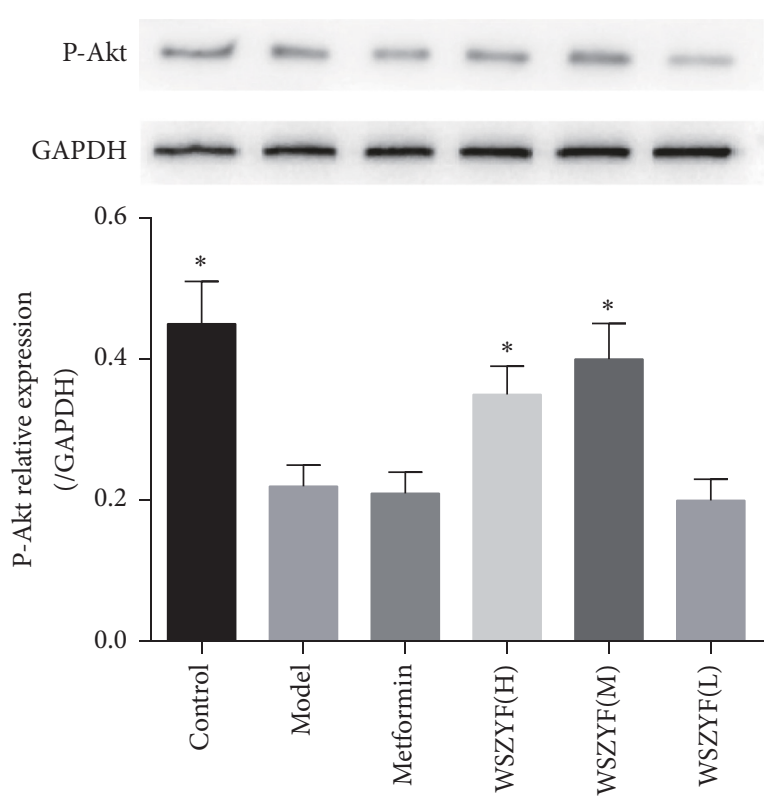

(b)
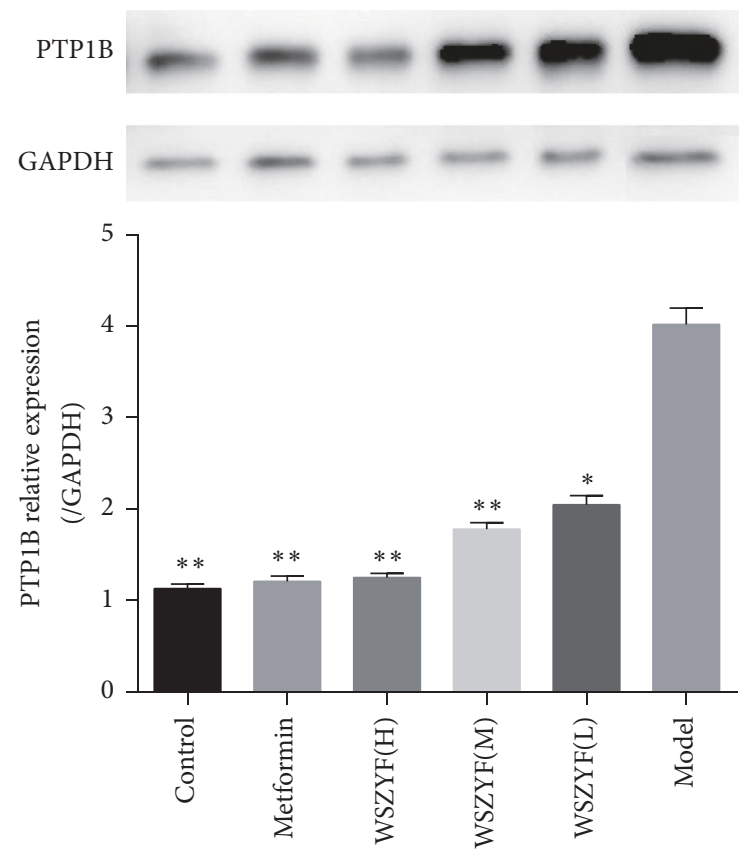

(d)

FIGURE 5: Effects of WSZYF on proteins related to insulin signal transduction. (a) P-IRS1 relative expression, (b) P-Akt relative expression, (c) GLUT4 relative expression, and (d) PTP1B relative expression. ${ }^{*} P<0.05$ and ${ }^{* *} P<0.01$ compared with model group.

Therefore, IRS-PI3K/Akt-GLUT4 signaling pathway exerts vital regulative effect in insulin signaling transduction of skeletal muscle. Any disorder in this pathway can reduce the sensitivity of skeletal muscles to insulin, resulting in impaired glucose uptake, utilization, glucose tolerance, and elevated blood glucose level [27]. Compared with control group, the expressions of p-IRS1, p-Akt, and GLUT4 decreased significantly in model group, indicating that insulin signaling transduction of skeletal muscle was abnormal and IR occurred.
With the treatment of WSZYF, expressions of the signal molecules mentioned above had been elevated, verifying that WSZYF improved IR via restoring insulin signaling transduction of skeletal muscle.

Protein tyrosine phosphatase-1B is an important target for T2DM treatment and has been proved to play a vital role in the negative regulation of insulin signaling transduction. It promotes the dephosphorylation of insulin receptor and IRS and downregulates insulin signaling transduction, ultimately 
leading to IR [13]. The expression of PTP1B increased in model group, thus inhibiting IRS1-Akt-GLUT4 signaling pathway and resulting in IR. This was similar to other studies [12, 14]. WSZYF reduced PTP1B expression in a dosedependent manner, followed by the restoration of IRS1-AktGLUT4 signaling pathway and mitigation of IR.

\section{Conclusion}

In summary, we investigated the antidiabetic effect of WSZYF in T2DM animal model and IR model of skeletal muscle in this study. The results demonstrated that WSZYF could regulate glucose mechanism and IR through modulating PTP1B-IRS1-Akt-GLUT4 signaling pathway. This study provides basis for further research on effective components in the treatment of T2DM.

\section{Conflicts of Interest}

The authors declare that they have no conflicts of interest regarding the publication of this manuscript.

\section{Authors' Contributions}

Chunyu Tian and Hong Chang contributed to the paper equally.

\section{Acknowledgments}

This paper was supported by the Natural Science Foundation of Hebei Province (H2015209025) and Scientific Research Program by Hebei Chinese Medicine Bureau (2016070).

\section{References}

[1] D. R. Whiting, L. Guariguata, C. Weil, and J. Shaw, "IDF Diabetes Atlas: global estimates of the prevalence of diabetes for 2011 and 2030," Diabetes Research and Clinical Practice, vol. 94, no. 3, pp. 311-321, 2011.

[2] P. Zimmet, K. G. M. M. Alberti, and J. Shaw, "Global and societal implications of the diabetes epidemic," Nature, vol. 414, no. 6865, pp. 782-787, 2001.

[3] American Diabetes Association, "Diagnosis and classification of diabetes mellitus," Diabetes Care, vol. 37, supplement 1, pp. S81-S90, 2014.

[4] B. He, M. Shi, and L. Zhang, "Beneficial effect of galanin on insulin sensitivity in muscle of type 2 diabetic rats," Physiology \& Behavior, vol. 103, no. 3-4, pp. 284-289, 2011.

[5] J. D. Best, S. E. Kahn, M. Ader, R. M. Watanabe, T.-C. Ni, and R. N. Bergman, "Role of glucose effectiveness in the determination of glucose tolerance," Diabetes Care, vol. 19, no. 9, pp. 1018-1030, 1996.

[6] F. Kanai, K. Ito, M. Todaka et al., "Insulin-Stimulated GLUT4 Translocation Is Relevant to the Phosphorylation of IRS-1 and the Activity of PI3 Kinase," Biochemical and Biophysical Research Communications, vol. 195, no. 2, pp. 762-768, 1993.

[7] N. Yu, X. Fang, D. Zhao et al., "Anti-diabetic effects of Jiang Tang Xiao Ke granule via PI3K/Akt signalling pathway in type 2 diabetes KKAy mice," PLoS ONE, vol. 12, no. 1, Article ID e0168980, 2017.
[8] F. Favaretto, G. Milan, G. B. Collin et al., "GLUT4 defects in adipose tissue are early signs of metabolic alterations in alms1GT/GT, a mouse model for obesity and insulin resistance," PLoS ONE, vol. 9, no. 10, Article ID e109540, 2014.

[9] A. Zorzano, M. Palacín, and A. Gumà, "Mechanisms regulating GLUT4 glucose transporter expression and glucose transport in skeletal muscle," Acta Physiologica Scandinavica, vol. 183, no. 1, pp. 43-58, 2005.

[10] X. Yang, J. Yang, C. Xu et al., "Antidiabetic effects of flavonoids from Sophora flavescens EtOAc extract in type 2 diabetic KK-ay mice," Journal of Ethnopharmacology, vol. 171, pp. 161-170, 2015.

[11] L. Yang, Z. Wang, L. Jiang, W. Sun, Q. Fan, and T. Liu, "Total flavonoids extracted from oxytropis falcata bunge improve insulin resistance through regulation on the IKKbeta/NFkappaB inflammatory pathway," Evidence-Based Complementary and Alternative Medicine, vol. 2017, Article ID 2405124, 6 pages, 2017.

[12] W. Ji, X. Chen, J. Lv et al., "Liraglutide exerts antidiabetic effect via PTP1B and PI3K/Akt2 signaling pathway in skeletal muscle of KKAy mice," International Journal of Endocrinology, vol. 2014, Article ID 312452, 9 pages, 2014.

[13] D. Patel, M. Jain, S. R. Shah et al., "Discovery of potent, selective and orally bioavailable triaryl-sulfonamide based PTP1B inhibitors," Bioorganic \& Medicinal Chemistry Letters, vol. 22, no. 2, pp. 1111-1117, 2012.

[14] Q.-C. Liu, T.-T. Guo, L. Zhang et al., "Synthesis and biological evaluation of oleanolic acid derivatives as PTP1B inhibitors," European Journal of Medicinal Chemistry, vol. 63, pp. 511-522, 2013.

[15] T. Wang and Y. Fan, "Effect of tetrahydroxy stilbene glucoside from Polygoni Multiflori Radix for insulin resistance in skeletal muscle of type 2 diabetes rats," China Medical Herald, vol. 13, no. 12 , pp. 25-28, 2016.

[16] C. Y. Yonamine, E. Pinheiro-Machado, M. L. Michalani et al., "Resveratrol improves glycemic control in type 2 diabetic obese mice by regulating glucose transporter expression in skeletal muscle and liver," Molecules, vol. 22, no. 7, p. 1180, 2017.

[17] F.-L. Hsu, C.-F. Huang, Y.-W. Chen et al., "Antidiabetic effects of pterosin A, a small-molecular-weight natural product, on diabetic mouse models," Diabetes, vol. 62, no. 2, pp. 628-638, 2013.

[18] X. M. Zhou, C. Y. Tian, X. J. La et al., "Effect of water extract of shizidaiping prescription on insulin resistance of skeletal muscle cells," Natural Product Research and Development, vol. 28, pp. 1139-1143, 2016.

[19] J. K. Prasain, N. Peng, R. Rajbhandari, and J. M. Wyss, "The Chinese Pueraria root extract (Pueraria lobata) ameliorates impaired glucose and lipid metabolism in obese mice," Phytomedicine, vol. 20, no. 1, pp. 17-23, 2012.

[20] K. Srinivasan and P. Ramarao, "Animal models in type 2 diabetes research: an overview," Indian Journal of Medical Research, vol. 125, no. 3, pp. 451-472, 2007.

[21] V. Saini, "Molecular mechanisms of insulin resistance in type 2 diabetes mellitus," World Journal of Diabetes, vol. 1, no. 3, pp. 68-75, 2010.

[22] K. Morino, K. F. Petersen, S. Dufour et al., "Reduced mitochondrial density and increased IRS-1 serine phosphorylation in muscle of insulin-resistant offspring of type 2 diabetic parents," The Journal of Clinical Investigation, vol. 115, no. 12, pp. 35873593, 2005.

[23] T. Shibata, A. Takaguri, K. Ichihara, and K. Satoh, "Inhibition of the TNF- $\alpha$-induced serine phosphorylation of IRS- 1 at $636 / 639$ 
by AICAR," Journal of Pharmacological Sciences, vol. 122, no. 2, pp. 93-102, 2013.

[24] A. Koh, M. N. Lee, Y. R. Yang et al., "C1-Ten is a protein tyrosine phosphatase of insulin receptor substrate 1 (IRS-1), regulating IRS-1 stability and muscle atrophy," Molecular and Cellular Biology, vol. 33, no. 8, pp. 1608-1620, 2013.

[25] M. H. A. Bakar, K.-K. Cheng, M. R. Sarmidi, H. Yaakob, and H. Z. Huri, "Celastrol protects against antimycin A-induced insulin resistance in human skeletal muscle cells," Molecules, vol. 20, no. 5, pp. 8242-8269, 2015.

[26] N. Sharma, D. A. Sequea, C. M. Castorena, E. B. Arias, N. R. Qi, and G. D. Cartee, "Heterogeneous effects of calorie restriction on in vivo glucose uptake and insulin signaling of individual rat skeletal muscles," PLoS ONE, vol. 8, no. 6, Article ID e65118, 2013.

[27] Q. Liu, X. Li, C. Li, Y. Zheng, G. Peng, and D. J. McPhee, "1deoxynojirimycin alleviates insulin resistance via activation of insulin signaling PI3K/AKT pathway in skeletal muscle of $\mathrm{db} / \mathrm{db}$ mice," Molecules, vol. 20, no. 12, pp. 21700-21714, 2015. 


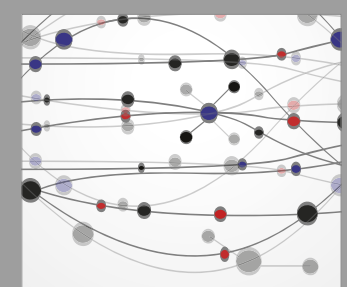

The Scientific World Journal
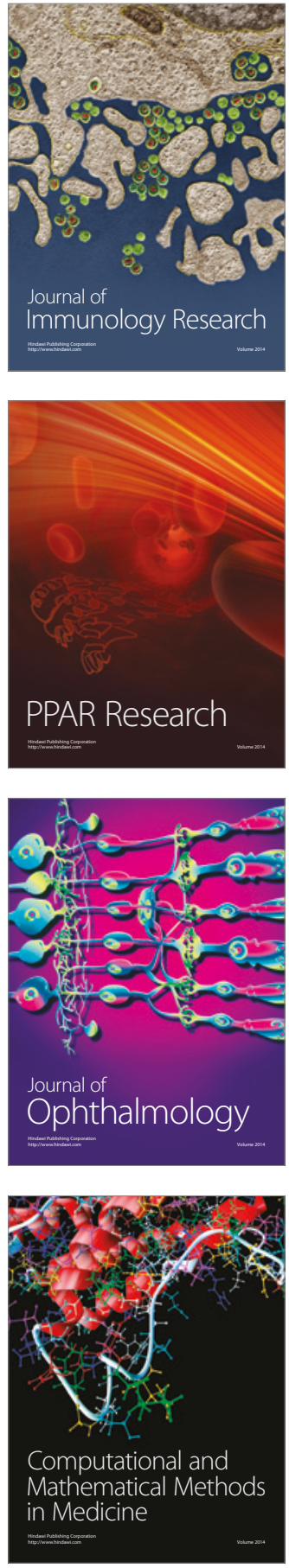

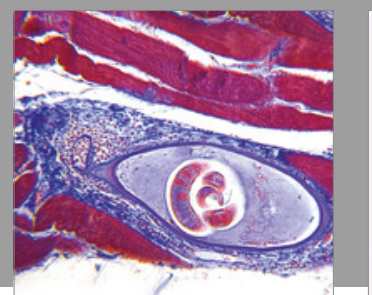

Gastroenterology Research and Practice
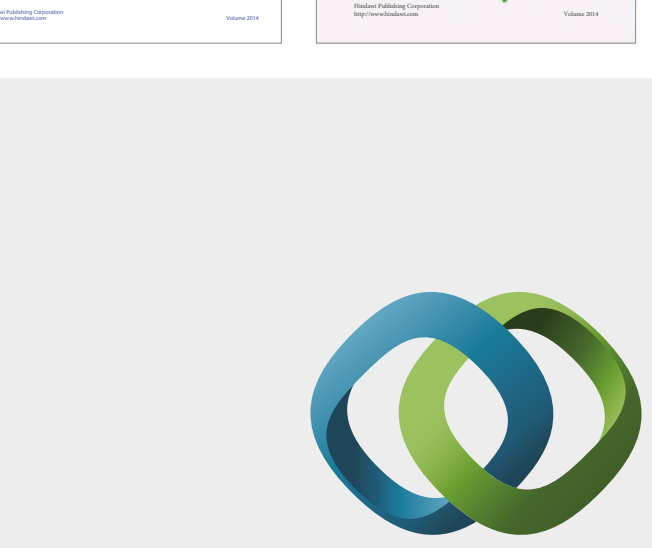

\section{Hindawi}

Submit your manuscripts at

https://www.hindawi.com
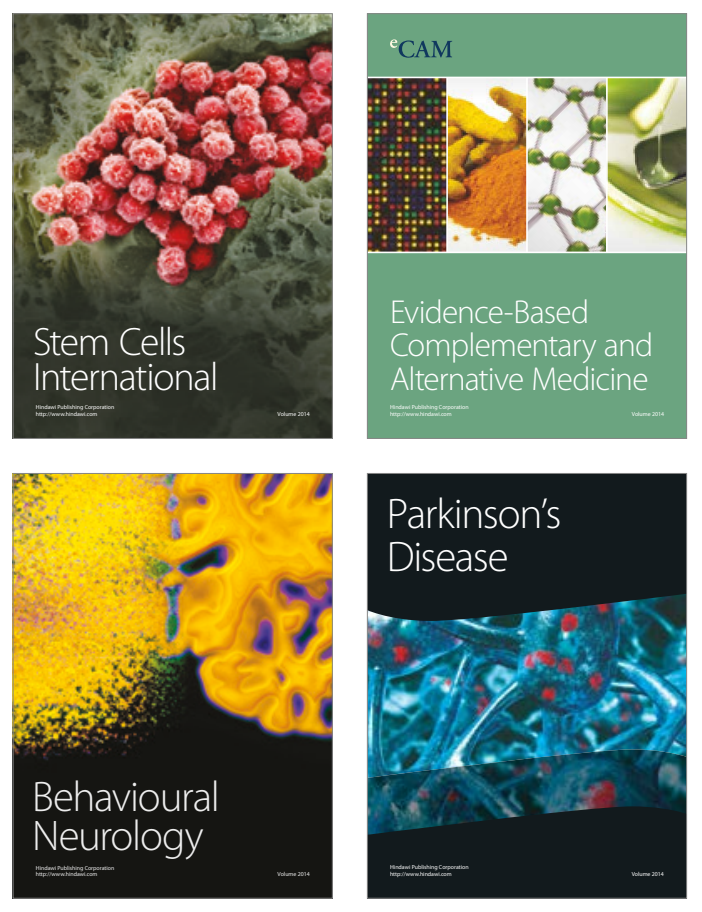
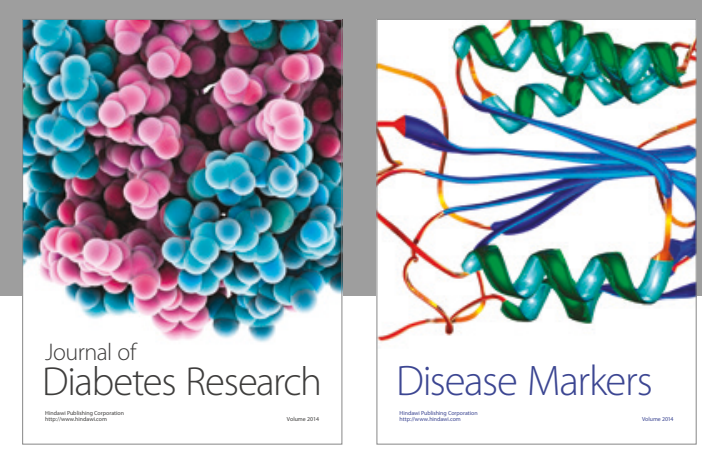

Disease Markers
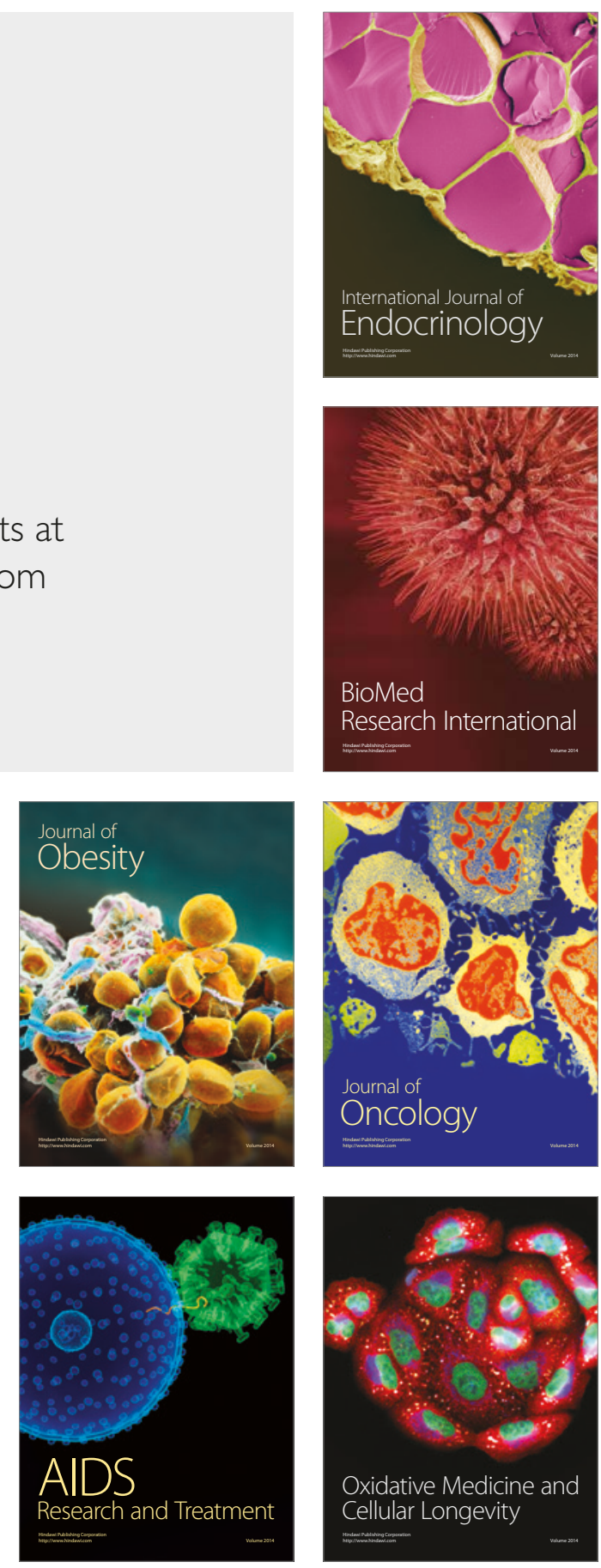